\title{
Progress of pulsed light sterilization technology in the food field
}

\author{
Weijin Fang ${ }^{1}$, Shengping Xue ${ }^{1, *}$, Yitong Yue ${ }^{2}$ \\ ${ }^{1,2}$ College of Bioscience and Engineering, Hebei University of Economics and Business, Shijiazhuang, 050061, Hebei, China
}

\begin{abstract}
In recent years, the rate of disease outbreaks caused by food-borne microorganisms is gradually increasing. The abuse of food preservatives has not only caused environmental problems, but also led to the gradual increase in the resistance of bacteria. As a non-thermal physical sterilization technology, pulsed light sterilization has attracted more and more attention in the field of food sterilization. Traditional heat sterilization will destroy the quality of food and cannot be used for preservation of fruits and vegetables. Chemical reagents will cause environmental problems. The pulsed light sterilization technology has the advantages of low cost, pollution-free, safe and efficient, and will not damage food quality. It can be perfectly applied in the field of food sterilization. Pulsed light can also be used in combination with other sterilization technologies. For example, the combined application of pectin coating and UV sterilization technology to treat food will achieve better results. The research on pulsed light sterilization at home and abroad is mainly based on the application in food sterilization. This article describes the mechanism of pulsed light sterilization, introduces the application examples of pulsed light sterilization in the food field, and discusses the impact on food quality.
\end{abstract}

\section{INTRODUCTION}

Food-borne diseases caused by eating food contaminated by microorganisms are still the main cause of morbidity worldwide $^{[1]}$. Therefore, adopting appropriate methods to reduce the hazards of food microorganisms is increasingly important for reducing the outbreak rate of foodborne diseases. Nowadays, there are a variety of methods for food preservation, such as heating, adding preservatives, freezing, and drying. These technologies can ensure the safety of food, but they all have certain limitations. For example, they are restricted by the objects to be processed. Heat sterilization cannot be used to preserve the freshness of fruits and vegetables. Preservatives cause environmental problems. Heating, drying and freezing cause changes in certain substances and affect food quality. [2] In this context, physical non-thermal sterilization technology has been rapidly developed. This type of technology can maintain the nutritional value and sensory quality of food to the greatest extent while ensuring food safety. Among these emerging technologies, promising applications mainly include high-voltage processing, pulsed electric fields, and pulsed strong light sterilization technology ${ }^{[3]}$.

The well-known physical sterilization technology, continuous ultraviolet (UV) and visible light, has a very good killing effect on microbial cells. In recent years, a new method of using high power electricity to generate pulsed light (PL) to sterilize and preserve food has been applied ${ }^{[4]}$. PL has a good sterilization effect on the surface of the food and the environment in which the food is made. The earliest application areas of PL sterilization technology are mainly the sterilization of medical devices and transparent drugs. With the improvement of this technology, its application becomes more and more extensive, and it gradually transitions to food sterilization and preservation ${ }^{[5]}$. Nowadays, research on PL sterilization is rapidly expanding, but there is still a gap between basic research and applied research on food sterilization.

This article mainly introduces the application of PL sterilization technology in the food field, discusses the combined application of PL sterilization technology and other sterilization technologies, and explores the sterilization mechanism, effectiveness and impact of PL on food quality.

\section{PL sterilization mechanism}

PL is a non-thermal physical food sterilization method that uses strong, short-time broad-spectrum pulses to kill microorganisms on the surface of food and packaging materials. The sterilization effect of PL depends on the intensity of light $\left(\mathrm{J} / \mathrm{cm}^{2}\right)$ and the number of pulses ${ }^{[6]}$. In some studies, irradiation is usually expressed in second, but there is no information about the number of pulses.

*Corrsponding author: E-mail: xsp6210@163.com. 
PL is produced by converting high-speed electronic pulses into short-time, high-peak energy pulses using engineering technology that is magnified several times. The system usually consists of three parts: a power supply, a pulse device and a lamp. The energy is stored in a highpower capacitor for a long period of time (a fraction of a second), and then released into a specially designed xenon lamp device in a shorter time (ns to ms). The high energy injected into the lamp produces intense light pulses, which are concentrated in the illuminated area, usually lasting several hundred milliseconds ${ }^{[7]}$. These lights include light with wavelengths in the UV to near-infrared spectrum. The wavelength range is $180-1100 \mathrm{~nm}$, which is about 20,000 times the intensity of sunlight hitting the earth's surface ${ }^{[8]}$.

The sterilization effect of PL increases with the increase of light intensity has been confirmed. The rich content of broad-spectrum UV light, short time, and high peak power in PL have a strong destructive effect on microorganisms ${ }^{[9]}$. The UV range contained in PL has a great influence on the sterilization effect. It has been confirmed that if the wavelength range below $320 \mathrm{~nm}$ UV is removed, PL will not have a lethal effect on microorganisms ${ }^{[10]}$. PL mainly destroys nucleic acids, and UV light in PL mainly destroys DNA. The photochemical action causes pyrimidine bases to form dimers in the DNA of bacteria, viruses and other pathogens, blocking DNA replication, and if there is not enough repair mechanism, it will lead to the death of microorganisms ${ }^{[11]}$.

Enzymatic repair of DNA does not occur after PL treatment, because the damage caused by PL is too severe for the repair mechanism to work ${ }^{[12]}$.

\section{Technical advantages}

\subsection{Comparison between PL sterilization and traditional thermal sterilization}

For traditional heat sterilization technology, because it can accurately kill various microorganisms, and the degree of sterilization can be accurately controlled, as an effective sterilization method, it is widely used in the food sterilization. However, heat sterilization requires high temperature conditions. It will inevitably affect the sensory, color, quality and nutrients of the food ${ }^{[13]}$. As a new type of non-thermal sterilization technology, PL sterilization technology not only has the advantages of energy saving, high efficiency and safety, but also can effectively kill microorganisms while maintaining the color, quality and taste of food to the maximum extent, effectively making up for the defects of traditional heat sterilization technology.

\subsection{Comparison between PL sterilization and UV sterilization}

The principle of UV sterilization is that UV rays can destroy the molecular structure of DNA or RNA in microbial cells and cause the death of bacteria, achieving the effect of disinfection and sterilization. Photochemical action is the main mechanism of traditional UV sterilization. For molds and spore microorganisms, it is difficult for UV to penetrate the dense cell wall structure, causing DNA (RNA) to be unable to absorb UV rays. PL sterilization not only relies on photochemical action, photothermal action and photophysical action also exert effects at the same time. When microorganisms receive PL at close range, they absorb a large amount of light energy in a short time, and the surface temperature of microorganisms will rise sharply. The structure will be completely destroyed and died ${ }^{[14]}$. Since the entire sterilization process lasts for a very short time, the irradiated object will not heat up, nor will it affect nutrients.

\section{PL sterilization effect on different microorganisms}

Table 1 Sterilization effect of different microorganisms by PL

\begin{tabular}{lcc}
\hline \multicolumn{1}{c}{ Strain } & Processing energy & Effect \\
\hline & & \\
Botrytis cinera & $15 \mathrm{~Hz}, 30 \mu \mathrm{s}$ & $3 \sim 4 \operatorname{logs}$ \\
L. Innocua & $12 \mathrm{~J} / \mathrm{cm}^{2}$ & $1.11 \operatorname{logs}$ \\
Salmonella enterica & $11.9 \mathrm{~J} / \mathrm{cm}^{2}$ & $1.8 \operatorname{logs}$ \\
L. Monocytogenes & $1.75 \mathrm{~J} / \mathrm{cm}^{2}$ & $2.2 \operatorname{logs}$ \\
E. coli O157:H7 & $28.2 \mathrm{~J} / \mathrm{cm}^{2}$ & $>5.7 \operatorname{logs}$ \\
Penicillium expansum & $32 \mathrm{~J} / \mathrm{cm}^{2}$ & $3.76 \operatorname{logs}$ \\
Pseudomonas aeruginosa $0.97 \mathrm{~J} / \mathrm{cm}^{2}$ & $7 \operatorname{logs}$ \\
Cryptosporidium parvum $6.29 \mu \mathrm{J} / \mathrm{cm}^{2}$ & $4 \operatorname{logs}$ \\
Murine orovirus & $14.02 \mathrm{~J} / \mathrm{cm}^{2}$ & $4.79 \operatorname{logs}$ \\
\hline
\end{tabular}

It can be seen from Table 1 that different microorganisms have different sensitivity to PL.Most food-borne microorganisms have been reduced by more than $3 \operatorname{logs}$ after PL treatment.It can also be seen from Table 1 that Gram-negative bacteria are less sensitive to PL than Gram-positive bacteria.

\section{Application fields}

\subsection{Application in liquid food}

The penetrating power of PL becomes weaker as the transparency of liquid food gradually decreases. For example, it has higher penetrating power to water, but it has weak penetrating power to liquid foods with higher turbidity such as milk products. In an aqueous solution, the lower the penetration of light, the worse the sterilization effect of PL. Smith ${ }^{[15]}$ used PL with a wavelength of $248 \mathrm{~nm}$ to process four sets of pre-set bulk milk at an intensity of $25 \mathrm{~J} / \mathrm{cm}^{2}$, and then observed them at $4^{\circ} \mathrm{C}$ and room temperature $\left(22^{\circ} \mathrm{C}\right)$ for 21 days. During this period of the two temperature conditions milk does not breed any bacteria. Fine ${ }^{[16]}$ et al. set up 1-25 pulse experiments to explore the PL intensity required to completely kill beer yeast, and found that the PL intensity corresponding to 24 pulses was $58.51 \mathrm{~J} / \mathrm{cm}^{2}$, which completely inactivated the microorganisms. By adjusting the distance between the light source and the experimental 
object to adjust the PL intensity, it is found that the microorganisms can be completely killed when the energy reaches $58 \sim 117 \mathrm{~J} / \mathrm{cm}^{2}$. Elmnasser ${ }^{[17]}$ and others explored whether the amino acid composition and UV absorbance of milk were changed after PL was used to treat milk. Experimental results confirmed that only a small amount of disulfide bond aggregation occurred after PL was used to treat milk. The structure of amino acids has not changed, and oxidation of fat has not occurred.

\subsection{Application in fresh keeping of fruits and vegetables}

PL keeps fruits and vegetables fresh through the following two aspects: (1) Extend the shelf life of fruits and vegetables. (2) Inhibit the browning of fruits and vegetables. When the amount of yeast in fruits and vegetables reaches $10^{5} \mathrm{CFUg}^{-1}$, decay and deterioration can be detected. The large water content, high sugar content, and low $\mathrm{pH}$ of fresh fruits and vegetables can easily cause the reproduction of microorganisms, leading to rot and economic losses. Many experiments have confirmed that PL has a significant effect in killing microorganisms on fruits and vegetables and extending their shelf life. Ripe blueberries have a short shelf life and are easily rotted by various pathogens. Cao ${ }^{[18]}$ explored the effect of PL on blueberry, such as the inactivation of Salmonella and the shelf life, quality attributes and beneficial compounds of blueberries. Set PL (untreated) $\left(6 \mathrm{~J} / \mathrm{cm}^{2}\right)$ and water-assisted PL (the sample is stirred in water during PL treatment; $9 \mathrm{~J} / \mathrm{cm}^{2}$ ), dry control (untreated) and water-assisted control (washed without PL), respectively. Subsequently, the blueberries were stored at room temperature ( 3 days) or $5^{\circ} \mathrm{C}$ ( 7 days). The experimental results confirmed that water-assisted PL treatment of blueberries can effectively remove Salmonella from blueberries, extend the shelf life of blueberries, and have almost no effect on the quality attributes and beneficial compounds of blueberries.

\subsection{Application in the field of meat preservation}

Many scholars have studied the impact of PL sterilization technology on meat quality and found that fresh chicken, fish and other meat products have a certain degree of adaptability to PL. Haughton et al. ${ }^{[19]}$ used high-intensity PL technology $(3 \mathrm{hz}, 505 \mathrm{~J} /$ pulse, $360 \mu \mathrm{s})$ to sterilize raw chicken and its related packaging materials. After $5 \mathrm{~s}$ of processing, Campylobacter jejuni, E. coli and Salmonella enteritidis the reductions were 1.22, 1.69 and $1.27 \operatorname{logs}$ on the material surface and contact surface respectively; the corresponding microbial reductions in skinless chicken breasts were $0.96,1.13$ and 1.35 logs. This experiment shows that PL is an effective decontamination method for meat surface and its packaging materials

\section{Combined application of PL sterilization technology and other technologies}

\subsection{PL and pectin coating technology to treat freshly cut fruits}

Moreira et al. ${ }^{[20]}$ soaked fresh-cut apples in an ascorbic acid/calcium chloride solution and then coated them with pectin, and then treated them with PL, which effectively reduce the softening and surface browning of apples. The pectin coating has no effect on the sensory and physical and chemical properties of apples. Pectin-coated PLtreated apples showed higher antioxidant activity value than PL-treated apples. At the end of storage, the combination of these two treatment methods resulted in a reduction of the number of microorganisms by nearly 2 logs. It is concluded that pectin-coated fresh-cut apples subjected to PL treatment can effectively maintain the quality of the fruit and extend its shelf life.

\subsection{Synergistic sterilization effect between PL and thermal effect}

B. cinere spores and $M$. fructigena spores are important reasons of economic loss during storage and transportation of strawberries and sweet cherries. David et al. ${ }^{[21]}$ first used a single PL to treat the two conidia, and applied $30 \mu \mathrm{s}$ pulse light treatment at a frequency of $15 \mathrm{hz}$. The time ranged from 1-250 s, and the maximum inactivation was 3 and $4 \operatorname{logs}$. Conidia inactivation increased with the increase of PL intensity, but did not reach complete inactivation. Two kinds of conidia were treated with heatlight pulse combination, and the spore inactivation effect was significantly increased. After $40 \mathrm{~s}$ PL treatment at $41^{\circ} \mathrm{C}$ for $15 \mathrm{~min}$, or at $41^{\circ} \mathrm{C}$ under $80 \mathrm{~s}$ light treatment for 10 min, it completely died.

\subsection{Synergistic sterilization effect between PL and UV}

Combining PL and UV-C $(\lambda=254 \mathrm{~nm})$ to sterilize strawberries inoculated with $B$. cinerea spores, it was found that this method did not significantly increase the sterilization effect on $B$. cinerea spores ${ }^{[21]}$. The reason may be that the sterilization mechanism of these two methods is similar, so the combined use may only increase their strength. And there is a DNA repair mechanism in the microorganism, which is triggered by UV irradiation . According to the current experimental research, the main object of PL sterilization is bacteria ${ }^{[9]}$, and the resistance of fungi to PL is higher than that of bacteria. Therefore, the use of PL technology to control fungal diseases of fruits and vegetables requires more experimental research.

\section{Conclusion and Prospect}

As a new type of cold sterilization technology, PL has the characteristics of high efficiency, environmental protection, and the ability to maximize the quality of food under certain conditions. PL has a very high level of killing microorganisms. For some opaque and irregular foods, although some microorganisms can be concealed, PL can still reduce the total number of microorganisms by $1 \sim 3$ logs. At the same time, the cost of using this 
technology to process food is not high, and it will not affect the quality of food, so the application prospect is very broad. In February 1994, the FDA accepted the application of PL to sterilize food. The application of PL in foreign countries mainly focuses on the sterilization of food and food packaging materials. However, there are few researches on PL sterilization technology in China, and it is in the initial stage, so more experiments are needed to further explore.

\section{Acknowledgements}

This work was financially supported by the Education department of Hebei Province Foundation (ZD2017229).

\section{References}

1. Elmnasser N,Guillou S,Leroi F,et al. Pulsed-light system as a novel food decontamination technology: a review[J]. Canadian Journal of Microbiology, 2007, 53(7): 813-821.

2. Vicente M. Gómez-López,Peter Ragaert,Johan Debevere,Frank Devlieghere. Pulsed light for food decontamination: a review $[\mathrm{J}]$. Trends in Food Science \&amp; Technology, 2007, 18(9): 464-473.

3. G.V. Barbosa-Cánovas. Nonthermal electrical methods in food preservation/Métodos eléctricos no térmicos para la conservación de alimentos[J]. Sage Publications, 1998, 4(5): 363-370.

4. Takeshita K,Yamanaka H,Sameshima $\mathrm{T}$, et al. Sterilization effect of pulsed light on various microorganisms[J].Journal of Antibacterial and Antifungal Agents,2002, (5): 277-284(in Japanese).

5. Gemma Oms-Oliu,Olga Martín-Belloso,Robert Soliva-Fortuny. Pulsed Light Treatments for Food Preservation. A Review[J]. Food and Bioprocess Technology,2010, 3(1): 13-23.

6. Wang T,MacGregor S J,Anderson J G,et al. Pulsed ultra-violet inactivation spectrum of Escherichia coli[J]. Water Research, 2005, 9 (13): 2921-2925.

7. Rowan N J,MacGregor S J,Anderson J G,et al. Pulsed light inactivation of food-related microorganisms[J]. Applied and Environmental Microbiology, 1999, 65 (3): 1312-1315.

8. Giese N,Darby J. Sensitivity of microorganisms to different wavelengths of UV light: implications on modeling ofmedium pressure UV systems[J]. Water Research,2000, 34(16): 4007-4013.

9. Slieman T A,Nicholson W L. Artificial and solar UV radiation induces strand breaks and cyclobutane pyrimidinedimers in Bacillus subtilis spore DNA[J]. Applied and Environmental Microbiology, 2000, 66 (1): 199-205.

10. Heinrich V,Zunabovic M,Varzakas T,Bergmair J,Kneifel W. Pulsed Light Treatment of Different
Food Types with a Special Focus on Meat: A Critical Review.[J]. Critical reviews in food science and nutrition,2016, 56(4): 591-613.

11. MacGregor S J,Rowan N J,Mcllvaney L,Anderson J G,Fouracre R A,Farish O. Light inactivation of foodrelated pathogenic bacteria using a pulsed power source.[J]. Letters in applied microbiology,1998, 27(2): 37-70.

12. Takeshita Kazuko,Shibato Junko,Sameshima Takashi,Fukunaga Sakae,Isobe Seiichiro,Arihara Keizo,Itoh Makoto. Damage of yeast cells induced by pulsed light irradiation.[J]. International journal of food microbiology,2003, 85(1-2): 151-158.

13. Kim Do-Kyun,Kang Dong-Hyun. Elevated Inactivation Efficacy of a Pulsed UVC LightEmitting Diode System for Foodborne Pathogens on Selective Media and Food Surfaces.[J]. Applied and environmental microbiology,2018, 84(20): e0134018.

14. D.H. Kingsley,R.E. Perez-Perez,G. Boyd,J. Sites,B.A. Niemira. Evaluation of 405-nm monochromatic light for inactivation of Tulane virus on blueberry surfaces[J]. Journal of Applied Microbiology, 2018, 124(4): 1017-1022.

15. Smith Wayne L,Lagunas-Solar Manuel C,Cullor James S. Use of pulsed ultraviolet laser light for the cold pasteurization of bovine milk.[J]. Journal of food protection, 2002, 65(9): 1480-1482.

16. Fine F,Gervais P. Efficiency of pulsed UV light for microbial decontamination of food powders.[J]. Journal of food protection, 2004, 67: 787-792.

17. Elmnasser N,Dalgalarrondo M,Orange N, et al. Effect of Pulsed-Light Treatment on Milk Proteins and Lipids[J]. Journal of Agricultural and Food Chemistry, 2008, 56: 1984-1991.

18. Cao Xinang,Huang Runze,Chen Haiqiang. Evaluation of pulsed light treatments on inactivation of Salmonella on blueberries and its impact on shelflife and quality attributes.[J]. International Journal of Food Microbiology, 2017, (260): 17-26.

19. Haughton P N, Lyng J G,Morgan D J,et al.Efficacy of high-intensity pulsed light for the microbiological decontamination of chicken,associated packaging, and contact surfaces[J].Food borne Pathogens \& Disease,2011, 8(1): 109.

20. Moreira María R,Álvarez María V,Martín-Belloso Olga,Soliva-Fortuny Robert. Effects of pulsed light treatments and pectin edible coatings on the quality of fresh-cut apples: a hurdle technology approach.[J]. Journal of the science of food and agriculture,2017, 97(1): 261-268.

21. Marquenie D,Michiels C W, Van Impe J F, et al.Pulsed white light in combination with UV-C and heat toreduce storage rot of strawberry[J]. Postharvest Biology and Technology,2003, 28(3): 455-461. 